\title{
Presença de acompanhantes em unidades de terapia intensiva pediátrica - revisão integrativa
}

\section{Presence of accompanyers in pediatric intensive therapy units} - integration review

\author{
João Victor Batista Cabral', Thais Patricia da Silva Carneiro², Ana Paula Sousa da Silva \\ 'Autor para correspondência. Universidade Federal de Pernambuco, Faculdades Integradas de Vitória de Santo Antão. Vitória de Santo \\ Antão, Pernambuco, Brasil. ORCID 0000-0001-8836-7875. jvbcabral@gmail.com \\ ${ }^{2}$ Faculdades Integradas de Vitória de Santo Antão. Vitória de Santo Antão, Pernambuco, Brasil. \\ thaispatricia21@hotmail.com, anap9887@gmail.com
}

\begin{abstract}
RESUMO | OBJETIVO: Descrever a importância de acompanhantes em unidades de terapia intensiva pediátrica. MÉTODO: Trata-se de revisão integrativa da literatura, com publicações entre os anos de 2000 a 2016 nas Bases de Dados da Biblioteca Virtual de Saúde. Foram incluídos artigos disponíveis eletronicamente, completos e em português. RESULTADOS: A presença do acompanhante é garantida pelo Estatuto da Criança e do Adolescente no Brasil e se mostra importante no que diz respeito à circunspeção da criança, como medida de segurança do cuidado, atenuante da aflição, ansiedade e medo, elemento de apoio psicológico e social, além da sua participação no delineamento do plano terapêutico. CONCLUSÃO: $O$ envolvimento dos familiares durante o processo de cuidar voltado para a criança hospitalizada e a garantia legal da presença do acompanhante no transcorrer de todo período de internação é vista como um grande progresso em termos de humanização, qualidade da assistência e minimização dos efeitos negativos ocasionados pela hospitalização.
\end{abstract}

DESCRITORES: Unidade de Terapia Intensiva Pediátrica. Hospitalização. Criança. Acompanhantes de pacientes. Família.

\begin{abstract}
I OBJECTIVE: To describe the importance of caregivers in pediatric intensive care units. METHOD: This is an integrative review of the literature, with publications between 2000 and 2016 in the Databases of the Virtual Health Library. Articles were included electronically, complete and in Portuguese. RESULTS: The presence of the companion is guaranteed by the Statute of the Child and Adolescent in Brazil and its is important in regard to the circumspection of the child, as a measure of safety of care, attenuating the affliction, anxiety and fear, element of support psychological and social, as well as their participation in the delineation of the therapeutic plan. CONCLUSION: The involvement of family members during the process of caring for the hospitalized child and the legal guarantee of the companion's presence during the entire hospitalization period is seen as a great progress in terms of humanization, quality of care and minimization of negative effects hospitalization.
\end{abstract}

DESCRIPTORS: Pediatric Intensive Care Units. Hospitalization. Child. Medical chaperones. Family. 


\section{Introdução}

O processo de hospitalização durante a infância traz para a família diversos sentimentos como estresse, aversão e receio e culpa, em virtude da responsabilização pela situação clínica da criança. $\mathrm{Na}$ proporção que o estado de saúde da criança se agrava e há necessidade de transferência para a unidade de terapia intensiva pediátrica (UTIP), normalmente manifestam-se sensações de espanto, incapacidade, frustração e medo, tanto para os pais, quanto para as crianças'.

Sabe-se que a internação é uma ocorrência crítica e difícil na vida de qualquer indivíduo, principalmente quando se refere à criança, uma vez que este processo pode acarretar diversas transformações em sua rotina diária do contexto familiar e muitas vezes interferir nos relacionamentos interpessoais ${ }^{2}$. A criança hospitalizada é exposta a situações desagradáveis em consequência da piora do seu estado de saúde, exacerbando sentimentos negativos relacionados à insegurança causada pela necessidade da realização de exames e procedimentos assistenciais; carreados de estresse, ansiedade e temor, devido à analogia imediata entre UTIP e morte, associada à separação temporária da família-criança ${ }^{3}$.

Os acompanhantes podem ser os genitores ou membros da família, com maioridade e responsabilidade legal pela criança. O Ministério da Saúde (MS) conceituou o acompanhante como um sujeito que possui total significância para a criança hospitalizada, sendo o mesmo um representador do seu contexto social que irá acompanhá-la no transcorrer de sua estadia no hospital ${ }^{2}$. O cuidar humanizado presume que $\circ$ profissional de saúde escute não apenas as principais queixas referidas pela criança, como também dê atenção ao seu acompanhante que, muitas vezes, se angustia, tem dúvidas sobre o tratamento e sofre com o medo de uma provável perda de seu ente querido, conforme a gravidade da doença, pois sua rotina doméstica e de trabalho é alterada em função da internação da criança e suas emoções podem estar afloradas, dificultando as relações no ambiente hospitalar ${ }^{4}$.

Deve-se ressaltar, que no ano de 1990 o Estatuto da Criança e do Adolescente (ECA), normatizou através dos artigos IX, XII e XIX que toda criança possui o direito da presença dos pais em período integral ao longo da hospitalização. Neste mesmo ponto de vista, a prerrogativa da participação dos familiares em companhia de seus filhos internados também foi preconizada pela Resolução no 41/95, do Conselho Nacional dos Direitos da Criança e do Adolescente (CONANDA), definindo que os cuidadores devem estar inseridos na assistência desde diagnóstico até o final do tratamento 5 .

A presença de um acompanhante, principalmente sendo da família e em especial a mãe, motiva e mantém uma relação interpessoal entre o profissional, a criança e respectivamente com seu cuidador, diminuindo os impactos negativos da separação familiar e dessa forma auxiliando a criança a se adaptar ao serviço de saúde, como também proporcionando uma melhor aceitação ao tratamento $e$ atenuando os motivos estressantes referentes à patologia, aos procedimentos e ao processo da hospitalizaçãó.

Sendo assim são notáveis os benefícios que o acompanhamento traz, minimizando o estresse da criança frente à internação, promovendo uma meIhor resposta ao tratamento. Mesmo sendo estabelecida pelo ECA a presença de um acompanhante para criança na internação, ainda é uma realidade pouco discutida e às vezes pouco compreendida nas instituições, principalmente nas UTI's. Levando em consideração as consequências no bem-estar biopsicossocial durante o período de hospitalização e das repercussões psíquicas para a criança, o presente estudo objetivou descrever a importância de acompanhantes em unidades de terapia intensiva pediátrica.

\section{Método}

Optou-se pela revisão integrativa da literatura para o método de investigação, objetivando o desígnio de buscar, avaliar, sumarizar e explicar a verificação prévia sobre o tema. As etapas percorridas para a operacionalização dessa revisão foram: 1 - escolha da questão norteadora; 2- seleção dos estudos compuseram a amostra a partir dos critérios de inclusão e exclusão da pesquisa; 3- estabelecimento das informações que serão captadas e classificação dos estudos; 4- julgamento analítico dos artigos inclusos na revisão; 5- análise crítica dos ar- 
tigos incluídos e discussão dos resultados; 6- relato da revisão e síntese das informações adquiridas no percorrer das outras etapas ${ }^{7}$.

A questão norteadora da vigente revisão constitui-se em: Qual a importância de acompanhantes em unidades de terapia intensiva pediátrica? A seleção dos estudos foi realizada pelos autores entre Agosto e Setembro de 2016, através do acesso online no portal da Biblioteca Virtual de Saúde (BVS), utilizando os seguintes descritores: Unidade de Terapia Intensiva Pediátrica; Hospitalização; Criança; Acompanhantes de Pacientes; e Família, descritores padronizados e presentes nos Descritores em Ciências da Saúde (DeCS).

A seleção dos artigos foi realizada através da associação dos descritores com combinação simultânea, realizada através do operador booleano "and": Unidade de Terapia Intensiva Pediátrica and Hospitalização and Criança and Acompanhantes de Pacientes and Família, obtendo 6.869 artigos, que após aplicação dos critérios de inclusão, resultaram em 06 estudos.
Para a seleção da amostra estabeleceu-se como critérios para a inclusão artigos disponíveis na íntegra eletronicamente, gratuitos, escritos no idioma português, publicados entre os anos de 2000 a 2016 e que respondessem à questão norteadora. Por sua vez, foram excluídos da pesquisa os artigos que não se encontrassem integral e gratuitamente disponíveis e os que não se enquadravam com o tema proposto.

Após a busca, foram realizadas leituras criteriosas das 06 publicações selecionadas para elaborar a presente revisão. Os resultados foram categorizados em um quadro ajustado para este propósito contendo os seguintes itens: título, autoria, objetivo, principais resultados e conclusão, conforme o quadro 2. A análise e interpretação dos resultados foram empreendidas em uma leitura exploratória com o objetivo de classificar e sintetizar as informações contidas nas fontes, de maneira que estas viabilizassem a aquisição de respostas ao problema do estudo.

Quadro 1. Seleção dos artigos incluídos no estudo.

\begin{tabular}{|c|c|c|c|c|c|}
\hline \multicolumn{6}{|c|}{ SELEÇÃO DOS ARTIGOS } \\
\hline $\begin{array}{c}1^{a} \text { Busca - Associação dos } \\
\text { Descritores }\end{array}$ & Critério 1 & Critério 2 & Critério 3 & Critério 4 & \multirow{2}{*}{$\begin{array}{l}\text { Artigos que se } \\
\text { enquadraram } \\
\text { com os critérios } \\
\text { estabelecidos }\end{array}$} \\
\hline $\begin{array}{l}\text { Unidade de Terapia Intensiva } \\
\text { Pediátrica and Hospitalização and } \\
\text { Criança and Acompanhantes de } \\
\text { Pacientes and Família. }\end{array}$ & $\begin{array}{l}\text { Disponível } \\
\text { Eletronicamente e } \\
\text { Gratuitamente }\end{array}$ & $\begin{array}{l}\text { Idioma - } \\
\text { Português }\end{array}$ & $\begin{array}{c}\text { Ano de } \\
\text { Publicação- } \\
2000 \text { a } \\
2016 \\
\end{array}$ & $\begin{array}{l}\text { Resposta à } \\
\text { questão } \\
\text { norteadora }\end{array}$ & \\
\hline 6.869 & 3.384 & 375 & 361 & 06 & 06 \\
\hline
\end{tabular}

Fonte: Autores, 2016.

Quanto às questões éticas, foram respeitados os princípios da honestidade e fidedignidade, bem como os direitos autorais e, em virtude da natureza bibliográfica da pesquisa, não houve necessidade de aprovação no Comitê de Ética em Pesquisa (CEP)

\section{Resultados}

Foram selecionados 06 estudos que atenderam aos critérios de inclusão instituídos. Em relação à contextualização com a temática abordada, os artigos selecionados foram publicados em 2007, 2009, 2012, 2013 e 2014, não sendo localizadas publicações referentes ao tema discutido nos demais anos e com os critérios estabelecidos. Os dados referentes ao quadro 2 apresentam as características dos artigos inclú́dos no presente estudo. 


\begin{tabular}{|c|c|c|c|c|}
\hline TÍTULO & AUTORES & OBJETIVOS & PRINCIPAIS RESULTADOS & CONCLUSÕES \\
\hline $\begin{array}{l}\text { A Percepção da } \\
\text { Familia sobre } \\
\text { sua Presença em } \\
\text { uma Unidade de } \\
\text { Terapia } \\
\text { Intensiva } \\
\text { Pediátrica e } \\
\text { Neonatal }\end{array}$ & $\begin{array}{l}\text { MOLINA RCM, } \\
\text { FONSECA EL, } \\
\text { WAIDMAN } \\
\text { MAP, } \\
\text { MARCON SS }\end{array}$ & $\begin{array}{l}\text { Compreender como as } \\
\text { familias percebem a } \\
\text { própria presença na } \\
\text { UTI e a aceitação } \\
\text { dessa presença por } \\
\text { parte dos profissionais } \\
\text { de saúde }\end{array}$ & $\begin{array}{l}\text { - Familia na UTI como base do } \\
\text { convivio social, unidade de } \\
\text { fortalecimento e segurança; } \\
\text { - Junto à família a criança sente-se: } \\
\text { segura, cuidada, animada, } \\
\text { acomodada, descansada, mais } \\
\text { confiante, alegre, amada, protegida, } \\
\text { tornando-se mais forte para } \\
\text { enfrentar a doença e a internação. }\end{array}$ & $\begin{array}{l}\text { Os pais sentem-se } \\
\text { mais tranquilos } \\
\text { quando permanecem } \\
\text { junto ao filho, pois } \\
\text { reconhecem que sua } \\
\text { presença, apoio e } \\
\text { carinho são essenciais } \\
\text { para a recuperação } \\
\text { da criança }\end{array}$ \\
\hline $\begin{array}{l}\text { Ação Intencional } \\
\text { do Familiar } \\
\text { Junto da } \\
\text { Criança em } \\
\text { Centro de } \\
\text { Terapia } \\
\text { Intensiva } \\
\text { Pediátrico }\end{array}$ & $\begin{array}{l}\text { CARDOSO } \\
\text { JMRM, } \\
\text { RODRIGUES } \\
\text { BMRD, } \\
\text { PACHECO, } \\
\text { STA, ARAÚJO, } \\
\text { BBM }\end{array}$ & $\begin{array}{l}\text { Apreender o que o } \\
\text { familiar tem em vista } \\
\text { quando permanece } \\
\text { com sua criança no } \\
\text { centro de tratamento } \\
\text { intensivo pediátrico }\end{array}$ & $\begin{array}{l}\text { - Oferta de sentimentos positivos; } \\
\text { - O cuidado, presença, se mostra } \\
\text { pelo estar - junto, por acompanhar, } \\
\text { envolver-se, comprometer-se, ter } \\
\text { preocupação com o outro; } \\
\text { - A intenção do familiar é não } \\
\text { somente fazer-se presente, como } \\
\text { também, cotidianamente acompanhar } \\
\text { a evolução clínica da criança. }\end{array}$ & $\begin{array}{l}\text { É necessário que os } \\
\text { enfermeiros } \\
\text { percebam o familiar } \\
\text { acompanhante como } \\
\text { um ser essencial ao } \\
\text { processo de cuidar, } \\
\text { contribuindo para } \\
\text { uma assistência } \\
\text { humanizada à criança } \\
\text { e sua família }\end{array}$ \\
\hline $\begin{array}{l}\text { Assistência em } \\
\text { unidade de } \\
\text { terapia intensiva } \\
\text { pediátrica: } \\
\text { percepção do } \\
\text { acompanhante }\end{array}$ & $\begin{array}{l}\text { RODRIGUES } \\
\text { EM, OLIVEIRA } \\
\text { ERC, JULIÃO, } \\
\text { AMSR }\end{array}$ & $\begin{array}{l}\text { Descrever a percepção } \\
\text { do acompanhante } \\
\text { sobre a assistência à } \\
\text { criança em unidade de } \\
\text { terapia intensiva } \\
\text { pediátrica }\end{array}$ & $\begin{array}{l}\text { - A visita e o acompanhante } \\
\text { estimulam a produção hormonal no } \\
\text { paciente, diminuindo o seu estado de } \\
\text { alerta e a ansiedade frente ao } \\
\text { desconhecido, trazendo mais } \\
\text { serenidade, confiança e, em } \\
\text { consequência, uma resposta mais } \\
\text { positiva aos tratamentos }\end{array}$ & $\begin{array}{l}\text { O estudo revelou que } \\
\text { as acompanhantes se } \\
\text { mostraram satisfeitas } \\
\text { em relação à } \\
\text { assistência que está } \\
\text { sendo prestada a sua } \\
\text { criança internada na } \\
\text { UTIP }\end{array}$ \\
\hline $\begin{array}{l}\text { Presença da } \\
\text { família nas } \\
\text { unidades de } \\
\text { terapia intensiva } \\
\text { pediátrica e } \\
\text { neonatal: visão } \\
\text { da equipe } \\
\text { multidisciplinar }\end{array}$ & $\begin{array}{l}\text { MOLINA RCM, } \\
\text { VARELA PLR, } \\
\text { CASTILHO AS, } \\
\text { BERCINI LO, } \\
\text { MARCON SS }\end{array}$ & $\begin{array}{l}\text { Compreender a visão } \\
\text { da equipe } \\
\text { multidisciplinar quanto } \\
\text { à presença da familia } \\
\text { nas Unidades de } \\
\text { Terapia Intensiva } \\
\text { pediátrica e neonatal }\end{array}$ & $\begin{array}{l}\text { - A presença da família, em especial } \\
\text { a da mãe, geralmente promove e } \\
\text { mantém a inter-relação } \\
\text { criança/familia/equipe, neutraliza os } \\
\text { efeitos negativos decorrentes da } \\
\text { separação, melhora sua adaptação } \\
\text { ao hospital, facilita a aceitação do } \\
\text { tratamento e ameniza os fatores } \\
\text { estressantes da doença, dos } \\
\text { procedimentos e da hospitalização }\end{array}$ & $\begin{array}{l}\text { Os relatos dos } \\
\text { profissionais } \\
\text { explicitaram o } \\
\text { conflito íntimo } \\
\text { vivenciado por cada } \\
\text { um com relação à } \\
\text { presença da família } \\
\text { na UTI neonatal ou } \\
\text { pediátrica, pois ao } \\
\text { mesmo tempo em que } \\
\text { sentem dificuldades } \\
\text { em inserir a família } \\
\text { no contexto da } \\
\text { unidade, reconhecem } \\
\text { o importante papel } \\
\text { desempenhado por } \\
\text { elas no processo de } \\
\text { hospitalização da } \\
\text { criança }\end{array}$ \\
\hline $\begin{array}{l}\text { Segurança da } \\
\text { Criança } \\
\text { Hospitalizada } \\
\text { na UTI: } \\
\text { Compreendendo } \\
\text { os Eventos } \\
\text { Adversos sob a } \\
\text { Ótica do } \\
\text { Acompanhante }\end{array}$ & $\begin{array}{l}\text { SILVA T, } \\
\text { WEGNER W, } \\
\text { PEDRO ENR. }\end{array}$ & $\begin{array}{l}\text { Descrever os eventos } \\
\text { adversos identificados } \\
\text { pelo familiar/cuida-dor } \\
\text { em uma UTIP }\end{array}$ & $\begin{array}{l}\text { - Percepção do familiar/cuidador } \\
\text { quanto: Falhas no processo de } \\
\text { comunicação; Prática não segura } \\
\text { referente à atuação profissional; } \\
\text { Requisitos para segurança do } \\
\text { paciente na UTIP }\end{array}$ & $\begin{array}{l}\text { Diante das } \\
\text { informações obtidas } \\
\text { pelo familiar/ } \\
\text { cuidador, percebeu- } \\
\text { se que: eventos } \\
\text { adversos foram } \\
\text { relacionados à } \\
\text { equipe de } \\
\text { Enfermagem; a } \\
\text { equipe médica, } \\
\text { negligencia o } \\
\text { familiar/cuidador } \\
\text { sobre a falta de } \\
\text { informação sobre o } \\
\text { estado de saúde da } \\
\text { criança hospitalizada. }\end{array}$ \\
\hline
\end{tabular}




\section{Discussão}

A busca e análise dos artigos incluídos neste estudo foram realizadas com o objetivo de identificar a temática em questão. A discussão, por analogia dos conteúdos abordados, ratificou-se em três categorias: processo de hospitalização, unidade de terapia intensiva pediátrica e a importância do acompanhante para a criança. Estas, por sua vez, foram executados de forma descritiva, conforme a seguir.

\section{Processo de Hospitalização}

O cuidado voltado à criança passa por diversas e significativas modificações em alguns países, especialmente após o século XIX, em consequência das alterações na assistência à saúde, na importância e relevância que a criança possui para a população e na atenção com os assuntos pertinentes à humanização durante o processo de cuidar. Para tal, foi necessário suceder mudanças na categorização da definição de criança, entendida como um ser humano em desenvolvimento, nos âmbitos psicossocial e físico ${ }^{8}$.

Com base nesse pressuposto, notou-se que no decorrer dos últimos anos houve um progresso notável nos cuidados direcionados à saúde, o que leva a ocasionar impacto na forma do indivíduo se relacionar com $\circ$ processo saúde-doença-hospitalização. Este processo é intensificado, quando está relacionado à criança, em virtude da dificuldade desta em se adaptar à nova rotina, bem como seus sentimentos são mais intensos e há uma maior proporção de alterações nas suas dimensões psicológica, física e social'.

A hospitalização é vista como algo que traz repercussões negativas para a vida da criança e de seus familiares, gerando preocupações e aflição. Logo, o processo de doença e hospitalização pode modificar o contexto intrafamiliar, propiciando sentimentos e emoções anômalas consequentes ao detrimento da rotina diária, das dúvidas referente sobre possibilidade de recuperar o equilíbrio e das incertezas sobre a nova realidade, visto que, apesar de aceitar o fato da internação, os indivíduos veem o hospital como um ambiente desconhecido em que não confiam ${ }^{10}$.
Portanto, o processo de hospitalização é caracterizado como um cenário complexo e muito difícil na fase da infância, uma vez que o mesmo remete modificações no cotidiano e no inter-relacionamento familiar, especialmente da mãe, que na maioria dos casos é a principal cuidadora da criança, ocasionando intensos sentimentos de ansiedade e angústia, levando a necessidade de uma assistência qualificada ${ }^{1}$.

Nesse sentido, a presença da doença e a internação, comumente são classificadas como as primeiras instabilidades enfrentadas na infância, tendo maior prevalência no decorrer dos primeiros anos de vida, resultante muitas vezes do nível socioeconômico e da práxis familiar, além de que, nesse período, estes indivíduos estão mais vulneráveis em virtude da fragilidade imunológica, sendo muitas vezes inábeis para enfrentar os fatores gênicos da doença ${ }^{12}$.

\section{Unidade de Terapia Intensiva Pediátrica}

No momento em que há agravamento do estado de saúde da criança e faz-se necessário o internamento da mesma em uma UTIP, esta circunstância gera mais desconforto para a família e acarreta sentimentos de aflição, medo, preocupação, descontentamento, debilidade, pânico, inquietação e dúvidas. $O$ desenvolvimento dessas emoções pode ser explicitado, devido ao fato de que a UTIP é designada ao atendimento de pessoas que apresentam o quadro clínico crítico ou semicrítico e necessitam de cuidados e terapêutica intensivos, executados através de uma equipe multidisciplinar ${ }^{13}$.

A UTIP é caracterizada como um setor fechado, ou seja, um ambiente com acesso restrito para os profissionais que atuam nela, possibilitando assim, a organização das ações desenvolvidas no processo de cuidar. A estrutura física e os dispositivos tecnológicos ali disponíveis manifestam cuidados e atenção a todos que entram nesse ambiente, agregada ao estado clínico dos pacientes, que geralmente são graves, e ao acentuado desempenho dos trabalhadores de saúde, em muitos casos tendem a se apresentá-la como um local hostil ${ }^{8,11}$.

Observa-se que a UTIP é um ambiente de alta complexidade, uma vez que esta possui uma rotina específica e múltiplos fatores estressores nas di- 
mensões psicológicas, físicas, ambientais e sociais. À vista disto, este setor pode revelar-se como um recinto temível e amedrontador tanto para $\circ$ paciente, quanto para seus familiares, especialmente pelo quantitativo de equipamentos ali dispostos?.

Com base nisso, nota-se que na maioria dos casos a UTIP é um setor apontando por algumas pessoas como um local hostil, pois o processo de hospitalização nessas unidades geralmente ocasiona traumas irremediáveis para o paciente e todos seus familiares, principalmente quando se refere à criança. Atribui-se a isso, o fato cultural de associação de que a internação neste ambiente remete à desventura, dor, sofrimento, fatalidade e morte ${ }^{11}$.

Dessa forma, na ocasião em que uma criança é hospitalizada em uma UTIP é imprescindível levar em consideração as modificações do contexto intrafamiliar decorrente da doença, pois a assistência da família é essencial para a aceitação e prosseguimento do tratamento e na colaboração desta com as normas e rotina do setor. Portanto, é de fundamental importância estimular o vínculo entre a criança, família e profissionais durante $\circ$ processo de hospitalização ${ }^{10}$.

\section{Importância do Acompanhante para a Criança}

No Brasil, em meados de 1980 deu-se início um movimento de estimulação da família na participação no cuidado com a saúde da criança internada. Rudimentarmente o estado de São Paulo iniciou a garantia do direito dos genitores no acompanhamento de suas crianças durante todo o processo de hospitalização, de acordo com a Resolução SS-165, publicada em 1988, que preconiza a importância do acompanhante 7 .

A partir disso, realizar assistência no âmbito da pediatria denota abranger não apenas a criança, como também, envolver suas dimensões relacionais, psicológicas e sociais, de tal maneira que se possa ponderar a criança e seus familiares como um paciente só ${ }^{2}$. Molina et al., (2009) ${ }^{1}$ descrevem a presença da família como base do convívio social e unidade de fortalecimento e segurança para a criança. Junto à família, a criança sente que está segura, cuidada, amada, protegida, mais confiante, - que a deixa fortalecida para o enfrentamento da doença e o período e ambiente de internação.
De acordo com Cardoso et al., (2013,) $)^{5}$ a inclusão e participação de um acompanhante no decurso da hospitalização, especialmente quando se refere a crianças internadas em UTIP, se mostra de extrema importância, no que diz respeito a maior demanda de circunspeção, pois nota-se que este processo pode ocasionar efeitos negativos para o paciente $e$ a presença de seus cuidadores pode ser um atenuante através da oferta de sentimentos positivos. $O$ cuidado, visto como "estar presente", se traduz no fato do acompanhante estar junto, acompanhar, envolver-se e se comprometer com a criança, além de acompanhar cotidianamente sua evolução clínica.

Em outro estudo realizado por Molina et al., $(2007)^{8}$, que avaliou a compreensão da equipe multidisciplinar quanto à presença da família na UTIP, foi visto que os profissionais consideram a presença da família, e em especial a da mãe, como uma forma de promover e manter a inter-relação criança/família/ equipe, tendo como resultados a neutralização dos efeitos negativos oriundos da separação e assim alcançando melhora da adaptação da criança ao hospital e facilitação da aceitação do tratamento.

Nesta vertente, o processo de cuidar envolvendo o acompanhante na UTIP, se constitui na sua inclusão na assistência aos seus filhos/acompanhados, como também na compreensão de que estes podem proporcionar apoio psicológico e social e serem agentes atuantes no delineamento do plano terapêutico com intuito de diminuir a ansiedade e o medo do processo de internação". A visita e o acompanhamento estimulam a produção hormonal do paciente e além de diminuírem a ansiedade, contribuem para um estado de serenidade e confiança, e como resultado, respostas mais positivas à terapêutica instituída ${ }^{8}$.

Embora o cuidado com saúde da criança na UTIP promovida pelos profissionais, seja eficiente e qualificado, o acompanhamento é indispensável, pois atenua a aflição e a ansiedade resultante da hospitalização. Contudo, é necessário que o cuidador seja instruído para colaborar com a assistência, sendo este orientado sobre os procedimentos de rotina que serão executados na criança, sua conduta no setor e a utilização dos materiais de proteção individual, assim tornando-o elemento de segurança durante sua estadia na unidade ${ }^{1}$. 
Silva, Wegner e Pedro (2012) ${ }^{14}$, em estudo sobre a segurança da criança hospitalizada em UTIP, verificaram que o familiar/cuidador descreveram a ocorrência de eventos adversos, destacando-se as falhas no processo de comunicação, práticas não seguras referentes à atuação dos profissionais, além dos requisitos de segurança para a criança durante a internação. Neste ínterim, é imprescindível assegurar o direito da assistência e atenção à saúde da criança e de seus cuidadores na UTIP, fundamentalmente pelos diversos aspectos envolvidos, na qual podem acarretar episódios desfavoráveis, afetando de modo direto na terapêutica e recuperação dos pacientes.

\section{Conclusão}

A permanência de um acompanhante no decorrer da internação é um direito garantido ao paciente através da legislação vigente do país, porém no que diz respeito à presença do cuidador no setor de UTIP a discussão ainda é fragilizada e parece ser pouco compreendida pelas instituições hospitalares.

Nesta perspectiva, o envolvimento do acompanhante durante $o$ processo de cuidar voltado para a criança hospitalizada e a garantia da sua presença no transcorrer de todo período de internação são vistos como progresso em termos de humanização, qualidade da assistência e minimização dos efeitos negativos ocasionados pela hospitalização.

O processo de hospitalização na infância retrata um evento difícil, com consequências profundas, que necessitam serem compreendidas em sua totalidade, assim a inclusão, acolhimento e participação do acompanhante garante a integralidade da assistência prestada à criança, com o propósito de atender suas necessidades físicas, psicológicas e sociais.

\section{Conflitos de interesses}

Nenhum conflito financeiro, legal ou político envolvendo terceiros (governo, empresas e fundações privadas, etc.) foi declarado para nenhum aspecto do trabalho submetido (incluindo mas não limitandose a subvenções e financiamentos, conselho consultivo, desenho de estudo, preparação de manuscrito, análise estatística, etc).

\section{Referências}

1. Molina RCM, Fonseca EL, Waidman MAP, Marcon SS. A percepção da família sobre sua presença em uma Unidade de Terapia Intensiva Pediátrica e Neonatal. Rev Esc Enferm USP. 2009;43(3):630-8. doi: 10.1590/S0080$\underline{62342009000300019}$

2. Faquinello P, Higarashi IH, Marcon SS. O Atendimento humanizado em unidade pediátrica: percepção do acompanhante da criança hospitalizada. Tex Cont Enf. 2007;16(4):609-16. doi: 10.1590/S0104$\underline{07072007000400004}$

3. Martins MS, Goese PB, Barrionovo MM, Massarollo MCKB. Direitos dos pacientes requeridos em um serviço público de ouvidoria. Rev Rene. 2015;16(3):337-44. doi: 10.15253/ rev\%20rene.v16i3.2771

4. Torquato IM, Collet NC, Dantas MS, Jonas MF, Trigueiro JVS, Nogueira MF. Assistência humanizada à criança hospitalizada: percepção do acompanhante. Rev Enferm UFPE On line. 2013;7(9):5541-9. doi: 10.5205/revol.352929105-1-SM.0709201323

5. Cardoso JMRM, Rodrigues BMRD, Pacheco STA, de Araújo BBM. Ação intencional do familiar junto da criança em Centro de Terapia Intensiva Pediátrico. Rev Enferm UERJ. 2013;21 (esp.1):600-5. doi: 10.12957/ reveri.2013.10033

6. de Oliveira GF, Dantas FDC, da Fonsêca PN. O impacto da hospitalização em crianças de 1 a 5 anos de idade. Rev SBPH. 2004;7(2):37-54.

7. Soares JD'AD, de Brito RS, de Carvalho JBL. A presença do pai/acompanhante no âmbito hospitalar: uma revisão integrativa. Rev Enferm UFPE On line. 2014;8(7):2095-106. doi: $10.5205 / \mathrm{revol} .5963-51246-1-\mathrm{RV} .0807201435$

8. Molina RCM, Varela PLR, Castilho SA, Bercini LO, Marcon SS. Presença da família nas unidades de terapia intensiva pediátrica e neonatal: visão da equipe multidisciplinar. Esc Anna Nery R Enferm. 2007;1 1(3):437-44. doi: 10.1590/ $\underline{\text { S1 } 414-81452007000300007}$

9. Gequelin J, Fernandes KF, Oliveira GP, Dyniewicz AM. Percepção de acompanhantes sobre a criança intubada em UTI pediátrica. Cogitare Enferm. 2014; 19(3):483-490. doi: $10.5380 /$ ce.v19i3.33434

10. Hayakawa LY, Marcon SS, Waidman MAP. A utilização do grupo como estratégia de suporte à mãe de criança internada em UTI pediátrica: relato de experiência. Rev Bra de Enf (Online). 2009;8(3). doi: 10.5935/1676$\underline{4285.20092661}$ 
11. Molina RCM, Higarashi IH, Marcon SS. Importância atribuída à rede de suporte social por mães com filhos em unidade intensiva. Esc Anna Nery Rev de Enf. 2014;18(1):6067. doi: $10.5935 / 1414-8145.20140009$

12. Rodrigues EM, Oliveira ERC, Julião AMS. Assistência em unidade de terapia intensiva pediátrica: percepção do acompanhante. $R$ Interd. 2014;7(4):39-49.

13. da Silva FR, Ballarin MLGS, Oliveira JC. Grupo de acompanhantes de crianças internadas em Unidade de Terapia Intensiva. Cad Ter Ocup UFSCar. 2015;23(4):871 877. doi: 10.4322/0104-4931.ctoRE0596

14. Silva T, Wegner W, Pedro ENR. Segurança da criança hospitalizada na UTI: compreendendo os eventos adversos sob a ótica do acompanhante. Rev Elet Enf. 2012;14(2):33744. doi: $10.5216 /$ ree.v1 4 i2. 12977 